\title{
Concept analysis of adherence
}

Chun-Mei Lyu, Li Zhang*

Department of Nursing, The First People's Hospital of Foshan, Affiliated to Sun Yat-Sen University, Foshan, Guangdong 528000, China

Received: 1 November 2018; Accepted: 25 December 2018; Published: 20 June 2019

Abstract: 0 bjective: To explore the concept of adherence in the context of rehabilitation of patients with chronic illnesses. This concept analysis is helpful in predicting health behaviors and intentions including physical activity and dietary behaviors in patients with chronic illnesses.

Methods: The framework of Walker and Avant was used to analyze the concept of adherence.

Results: Adherence is defined as the extent to which a person's behavior, such as taking medication, following a diet, and/or executing lifestyle changes, corresponds to agreed recommendations from a health-care provider. The antecedents of adherence are the biomedical status of the individual, social support, self-efficacy, and education. Self-report questionnaires and patient self-reporting are the most common measurement tools of adherence. Thus, the reliable and valid instrument of monitoring adherence in the clinical settings is challenging.

Conclusions: Analyzing the concept of adherence is necessary to help understand how best to promote adherence to improve healthrelated outcomes.

Keywords: adherence $\bullet$ rehabilitation of patients with chronic illnesses $\bullet$ the Theory of Planned Behavior • concept analysis

(c) Shanxi Medical Periodical Press.

\section{Introduction}

Adherence affects patient care and plays an important role in nursing practice. ${ }^{1}$ In Merriam-Webster's online dictionary, ${ }^{2}$ adherence is described as "to stick to something; to attach firmly to something." Taber's cyclopedic medical dictionary notes adherence as "the extent to which a patient's behavior coincides with medical advice."3 As reported by World Health Organization, adherence is defined as "the extent to which a person's behavior, such as taking medication, following a diet, and/or executing lifestyle changes, corresponds with agreed recommendations from a health-care provider." Compliance is often interchangeable when used with adherence to the report or the literature. Therefore, the definition of compliance must also be mentioned together. In general, compliance is used to describe a patient performing his or her behavior on medical recommendations. Thus, adherence is nonjudgmental and defines a mutual desire to achieve a specific behavior. ${ }^{4}$ It is much more comprehensive than compliance in describing a shared decision-making process to continue with medical recommendations. ${ }^{5}$ Nowadays, adherence has been used more frequently

How to cite this article: Lyu C-M, Zhang L. Concept analysis of adherence. Front Nurs. 2019; 2: 1-6.

* Corresponding author.

E-mail: 13927282790@163.com (L. Zhang).

Ә Open Access. @ 2019 Chen-Mei Lyu and Li Zhang, published by Sciendo. 
than compliance. As a multidimensional concept, adherence has been widely used in the health care system. ${ }^{6}$ With the rapid growth of the elderly population, chronic health problems have become a threat to daily life. Jankovic et al. ${ }^{7}$ found that greater adherence is linked to greater longevity. Adverse effects such as high blood pressure, resistance, toxicity, and intense relapses can occur in response to poor adherence. ${ }^{6}$ Despite these risks, in developing countries, only $50 \%$ of patients who are diagnosed with chronic illnesses follow the recommended treatment. ${ }^{8}$ Poor adherence results in reducing the effectiveness of treatment, threatens patient safety, increasedmorbidity, and medical financial cost. ${ }^{9}$ Nurses have the closest contact with patients; thus, they play an essential role in ensuring the patient adherence to longterm therapies. Improving adherence can increase the effect of treatment, develop clinical practice, decrease medical costs, and enhance patient safety.

\section{Methods}

The concept analysis is based on the Walker and Avant's concept analysis framework. ${ }^{10}$ This framework is widely used in the analysis of nursing-related concepts. It includes the following steps: selecting a concept, determining the aims or purpose of analysis, determining the defining attributes, constructing a model and contrary case, identifing antecedents and consequences, and defining empirical referents.

\section{Results}

\subsection{Defining attributes}

After literature review, four attributes of adherence that have been identified in the literature are self-efficacy, self-determination, autonomy, and relapse prevention.

\subsubsection{Self-efficacy}

Self-efficacy improvement plays an important role in the successful treatment of many chronic illnesses. Adam and Folds described self-efficacy as an individual's belief. ${ }^{9}$ It is an individual's internal intension to meet the desired goal. The patient's self-efficacy may affect their behavior and disease process. Self-efficacy is very important for the patient to adjust to a disability, especially in the area of chronic illness such as blood pressure control, glycemic control, and rehabilitation. ${ }^{11}$ For type 2 diabetes patients, self-management behaviors include blood glucose monitoring, weight management, medication administration, foot care, low sodium diet, and so on, which need great self-efficacy. If patients feel better, they are likely to adhere to the treatment. Positive strategies for self-efficacy interventions such as social support are very useful. The support may come from health-care providers, family, partners, and friends and is vital in helping patients manage and cope with the disease effectively. However, social support cannot be the only method in enhancing self-efficacy or adherence. Adam and Folds ${ }^{9}$ suggested that coaching should combine with counseling. Providing relevant knowledge and taking courses about how to cope with the disease can be effective to meet the treatment goals.

\subsubsection{Self-determination}

Self-determination relates to human motivation and personality. It is defined as the personal decision to do something. Comparing with external influence, selfdetermination is more dependent on inherent growth tendencies such as psychological needs. Recent research by Pearson noted that self-determination created a high level of adherence in specific individuals' behavior. ${ }^{12}$ For patients with chronic illness, health outcomes depend on voluntary actions and engagement. Edmunds et al. ${ }^{13}$ examined the differences in self-determination motivation. The study found that self-determination appeared to have a significant effect on individuals' psychological processes and influence exercise engagement. Some psychological needs, such as the need for autonomy, competence, and relatedness, were fulfilled. ${ }^{13}$ When patients feel satisfied, they would have a positive attitude and regulate their behavior well. In addition, self-determination forms motivational regulation to guide and adapt behavior for well-being. In contrast, less self-determination motivation and psychological dissatisfaction lead to poor outcomes.

\subsubsection{Autonomy}

Finocchario-Kessler et al. ${ }^{14}$ found that patient autonomy influenced beliefs, coping styles, and practices for patients' treatment adherence. Similarly, Williams et al. ${ }^{15}$ illustrated that autonomous regulation led to positive health behaviors and longer-term behavior change. Autonomous regulation is considered an internal perceived control (e.g., beliefs and decisionmaking), rather than the external control (such as persuasion or motivation from others). With the support of health-care providers, family, and friends, patients can foster autonomous regulation and enhance treatment adherence. ${ }^{15}$

\subsubsection{Relapse prevention}

There are many reasons why people cannot complete a task or meet the desired goal: "I do not have time" is one 
of the most common reasons. Thompson and McCabe found that relapse prevention is associated with adherence. ${ }^{16}$ Persistent poor adherence could make the overall health condition worse, because the patients would respond less to the medical treatment. They found that behavioral plans could improve from education about the illness. This can range from simply providing information on treatment or medication to enhancing adherence. Education can lead to early detection of warning signs and prevention of progression into full episodes. It has been reported that interventions that educate patients about their illness can lead to greater adherence and long-term outcomes. ${ }^{17}$

\subsection{Definition}

Adherence is defined as achieving self-determination and autonomy characterized by self-efficacy promotion and relapse prevention.

\subsection{Model case}

Mary was a 68-year-old woman, who was diagnosed with diabetes and hypertension. She went to hospital due to high blood pressure. The nurse checked her blood pressure, and it was $179 / 94 \mathrm{mmHg}$. Mary met with a doctor, Tom, and discussed options. Doctor Tom and Mary agreed that she should take a blood pressure medication to control the hypertension. In addition, some lifestyle changes (e.g., low-sodium dietary and more physical exercise) needed to be made to help her control hypertension. Mary followed the medication recommendations, and she came back to the hospital after 2 weeks to have her blood pressure and glucose levels checked. During the visit, Mary told Tom that she had been taking her medication as ordered and had started a new exercise program in her daily life. Her friends asked her go out to eat some McDonald's highsodium food, but she refused and kept eating a lowsodium diet. Mary's blood pressure was150/82 $\mathrm{mmHg}$. and she had lost $1.12 \mathrm{~kg}$ over the past 2 weeks. After 1 month, Mary went to the hospital for another followup visit. She reported that she was still eating a lowsodium diet, taking the medication, and doing exercise. Her blood pressure was $124 / 73 \mathrm{mmHg}$, and she had lost $1.81 \mathrm{~kg}$ over the past month. Mary was encouraged and told the doctor that she was going to continue taking the medications and maintain a healthy lifestyle to control her blood pressure and glucose levels.

\subsection{Contrary case}

Bob was a 76-year-old man who was diagnosed with diabetes and hypertension about 6 years ago. When he went to hospital, his blood pressure was $181 / 97 \mathrm{mmHg}$. In the past, he had been prescribed blood pressure medication, but he failed to take the medication. Tom and Bob discussed the treatment options, and Bob seemed totally unconcerned with what the doctor said. Tom outlined the disadvantage of uncontrolled hypertension, and Bob showed his understanding and agreed to begin taking it. Tom told Bob that he needed to come to the hospital for a follow-up visit within the next week; however, Bob went to the hospital about 2 weeks later. During the visit, He told Tom that he had been administrating the medication as he had recommended. Tom checked his blood pressure, and it was $175 / 85 \mathrm{mmHg}$. Tom increased the dosage of medication and told Bob to come back in 2 weeks. Bob did not come to the hospital until 2 months later. He was sent to the Emergency Department because of the respiratory infection and heart failure. His blood pressure was $181 / 89 \mathrm{mmHg}$, and he never took any medication to control his blood pressure. When Tom asked why he did not take the medication, he just told the doctor that he did not like how the medication made him feel.

\subsection{Antecedents}

The antecedents of adherence are the biomedical status of the individual, social support, self-efficacy, and education. Boyette et al. ${ }^{18}$ claimed that adherence for the elderly people in exercise was limited by biomedical status, socioeconomic status, education , and past personal experience in exercise. In contrast, they noted that gender, age, and occupation did not impact adherence. Olowookere et al. ${ }^{19}$ noted that adherence was influenced by levels of education, physical environment, physician influence, and living status. This study found that poor adherence was especially high in newly diagnosed patients. When patients lack information on their illness, they feel more afraid of exercise engagement. Good education is associated with higher adherence, which can reduce the possibility of life-threatening complications (e.g., stroke and cardiac failure). Second, patients without family and social support had equally poor drug and exercise adherence, because caregivers and family members can help remind these patients to adhere to the medical recommendations. Fitzpatrick et al. ${ }^{4}$ identified self-motivation and physical selfefficacy as keys to adherence. They also thought that physical environment and physicianinfluence played an important role in adherence. Elderly people will follow an exercise program if they think that the exercise will improve their sleeping, strength, or overall health condition. On the other hand, negative outcome expectations, along with the fear of pain and safety risks, impacted adherence. 


\subsection{Consequences}

Good adherence to treatment recommendations enhances patient's safety. It plays an essential role in the treatment of disease. Fitzpatrick et al. claimed that adherence promotes improved mental, physical, and social health and self-efficacy. ${ }^{4}$ Good adherence can promote healthy lifestyles such as not smoking, diet change, and increased physical activity. Furthermore, adherence influences disease treatment interventions and relapse prevention. Chawla et al. ${ }^{20}$ noted that improving adherence might be the lower cost and effective investment for reducing complications and disability, decreasing mortality and length of stay, and improving patients' quality of life. The most important of all, its improved health outcomes can be a positive physical and social effect for the patients. This positive psychological effect is impacted by adherence. In contrast, poor adherence could lead to an increased risk of dependence, toxicity, rebound effect, and developing resistance to therapies; Roebuck et al. ${ }^{21}$ stated that almost half of all Americans live with at least one chronic disease. Continuing the use of prescribed medications and following the medical recommendations are a key component for chronic diseases. Patients who adhere to the medical recommendations and exercise treatments experience a higher quality of life and better outcomes. As a result of this, some urgent care and inpatient hospital services would be used less.

\subsection{Empirical referents}

During the literature search, several measurement tools were used by nurse researchers in five studies of adherence from 2006 to 2016 . Clark et al. ${ }^{22}$ found that selfreport questionnaires and patient self-reporting were the most common measurement tools of adherence. It can provide an effective way of screening patients for nonadherent behavior and is easy for health-care providers to use. Physiological parameters, illness perceptions, and treatment perceptions can also be used in the evaluation of treatment recommendations of adherence in the selfreport questionnaires. Owing to the physiological factors (e.g., self-regulatory, biomedical, cognitive, behavioral, and communicative), underlying adherent behavior was complex; the study still had some low-risk participant bias in the measurement process. Furthermore, the reliable and valid instrument of monitoring adherence in the clinical settings is challenging. Electronic monitoring devices are also used to monitor medication adherence, and pill counts can be utilized. Medication event monitoring system can be used to access containers through a unique sensor on the lid of the containers and collect the data. ${ }^{23}$ Adherence may also be evaluated through the assessment of behaviors in the follow-up care. ${ }^{22}$ Milchak et al. explored JNC 7 adherence measurement tool of hypertension care; ${ }^{24}$ there are three treatment dimensions including laboratory, comorbidities, and follow-up intensity. Nevertheless, new tools should be developed, and the reliability of measurement tools must continually be evaluated.

\subsection{The Planned Behavior Theory applied in adherence}

The Theory of Planned Behavior is described as middle range theory. It can help people predict and explain an individual's behavior. Attitudes, perceived behavioral control, subjective norms, and behavioral intension are four main constructs of the Planned Behavior Theory. Attitude refers to negative or positive evaluations of the behavior (e.g., treatment recommendations of adherence are good for the patient's health condition); subjective norm refers to perceptions of pressure from others to perform the behavior (e.g., family members, partners, and friends would remind patient to perform treatment recommendations); and perceived behavioral control refers to judgment of whether it is easy or difficult to perform the behavior(e.g., it is easy for the patient to adhere to the treatment recommendations).

The Theory of Planned Behavior provides a framework to predict why patients change their behavior. The theory is also applied in predicting health behaviors and intentions including physical activity and dietary behaviors in patients with acute and chronic illnesses. ${ }^{25}$ Sabaté stated that adherence in chronic illness requires voluntary actions and engagement, ${ }^{6}$ because it is a long-term commitment of managing the disease by following the treatment recommendations by the health-care provider. For instance, exercise is easy to do and benefit health. People hold the attitude that "Adherence behavioral pattern is unnecessary for me." or "I don't need to do it." Even though regular exercise is good for health condition, people are unlikely to stick to prescribed exercise unless they have a diagnosed disease (e.g., hypertension and diabetes). That being said, when an individual is diagnosed with a cardiac disease, nonadherence is more serious than simply not undertaking a healthy behavior. If the patient does not adhere to the treatment recommendations, their health condition might deteriorate rapidly. ${ }^{25}$ Behavior is determined by a person's beliefs. Armitage and Conner noted that "the role of intention as a mediator of the effect of attitudes and subjective norms on behavior, ${ }^{26}$ and perceived behavioral control as a predictor of both intention and behavior." Rich ${ }^{24}$ noted the effectiveness of the Planned Behavior Theory for predicting adherence to treatment recommendations in chronic illnesses across 
multiple studies. The study found that the Theory of Planned Behavior can explain the variance in intention and adherence behavior. Gipson and King showed that the Theory of Planned Behaviorhas been applied to predict the adherence of patients to change their health behavior successfully. ${ }^{27}$

\section{Conclusions}

Adherence is a phenomenon that is essential for healthcare providers and creates an opportunity to develop interventions to improve patients' outcomes. Identification of antecedents, attributes, and consequences of adherence helps health-care providers understand the relation between practice and research. This will improve the knowledge of how the concept is embedded in the Theory of Planned Behavior. As mentioned earlier, many studies have showed that the Theory of Planned Behavior has been widely applied to predict the adherence of patients to change their health behavior. Healthcare providers, friends, and family are vital in helping patients manage and cope with the disease effectively. Owing to the unique characteristics of adherence behaviors, the reliability and validity of measurement tools for predicting adherence must continually be reevaluated. Self-reported questionnaires and follow-up care are still considered the main measurement tools and examining methods. The collected data are dynamic, not static. Patients can change their minds under different conditions, including emotional change and behavior change. New tools should be developed, especially some that reflect the social and psychological context. Further studies are still needed to assess the effectiveness of other theories in predicting adherence behavior as well as the evaluation and development of adherence interventions.

\section{Highlights}

Adherence is defined as the extent to which a person's behavior-taking medication, following a diet, and/or executing lifestyle changes, corresponds with agreed recommendations from a health-care provider. This study analyzed the concept of adherence in the context of rehabilitation of patients with chronic illnesses. From reviewing previous literatures, four attributes of adherence are identified as follows: self-efficacy, selfdetermination, autonomy, and relapse prevention. The presentation of a model case and a contrary case has shown the differences related to attributes between effective and invalid adherence. The Theory of Planned Behavior provides a framework to predict why patients change their behavior. The theory is also applied in predicting health behaviors and intentions including physical activity and dietary behaviors in patients.

\section{Acknowledgments}

This study was completed during the first author's study in Ireland. The authors appreciate the suggestions and comments provided by Professor Gerardina Harnett in the Institute of Technology, Tralee.

\section{Conflicts of interest}

All contributing authors declare no conflicts of interest.

\section{References}

1. Gardner CL. Adherence: a concept analysis. Int Nurs Knowl. 2015; 26:96-101.

2. Merriam. Merriam-Webster's dictionary online, 2013. America: Merriam-Webster. http://www. merriam-webster.com/. Accessed January 29, 2018.

3. Venes D. Taber's Cyclopedic Medical Dictionary (22nd ed.). Philadelphia: F. A. Davis Company; 2013.

4. Fitzpatrick JJ, McCarthy G., RNYT R, eds. Nursing Concept Analysis: Applications to Research and Practice. Springer; 2016.

5. Van Dulmen S, Sluijs E, Van Dijk L, De Ridder D, Heerdink R, Bensing J. Patient adherence to medical treatment: a review of reviews. BMC Health Serv Res. 2007;7:1.

6. Sabaté E. Adherence to long-term therapies: evidence for action. World Health Organisation; 2003.
7. Jankovic N, Geelen A, Streppel MT, et al. Adherence to a healthy diet according to the World Health Organisation guidelines and all-cause mortality in elderly adults from Europe and the United States. Am J Epidemiol. 2014;180:978-988.

8. AlHewiti A. Adherence to long-term therapies and beliefs about medications. Int J Family Med. 2014;2014:479596.

9. Adam J, Folds L. Depression, self-efficacy, and adherence in patients with type 2 diabetes. $J$ Nurs Pract. 2014;10:646-652.

10. Al-Khawaldeh OA, Al-Hassan MA, Froelicher ES, Self-efficacy, self-management, and glycemic control in adults with type 2 diabetes mellitus. J Diabetes Complic. 2012; 26:10-16.

11. Pearson ES. The 'how-to' of health behaviour change brought to life: a theoretical analysis of the 
Co-Active coaching model and its underpinnings in self-determination theory. Coaching. 2011;4: 89-103.

12. Edmunds J, Ntoumanis N, Duda JL. Adherence and well-being in overweight and obese patients referred to an exercise on prescription scheme: a self-determination theory perspective. Psychol Sport Exerc. 2007;8:722-740.

13. Finocchario-Kessler S, Catley D, Berkley-Patton $\mathrm{J}$, et al. Baseline predictors of ninety percent or higher antiretroviral therapy adherence in a diverse urban sample: the role of patient autonomy and fatalistic religious beliefs. AIDS Patient Care STDs. 2011;25:103-111.

14. Williams GG, Gagné M, Ryan RM, Deci, EL. Facilitating autonomous motivation for smoking cessation. Health Psychol. 2002;21:40.

15. Thompson L, McCabe R. The effect of clinicianpatient alliance and communication on treatment adherence in mental health care: a systematic review. BMC Psychiatr. 2012;12:1.

16. Bond K, Anderson IM. Psychoeducation for relapse prevention in bipolar disorder: a systematic review of efficacy in randomized controlled trials. Bipolar Disorders. 2015; 17:349-362.

17. Boyette LW, Lloyd A, Boyette JE, Watkins E. Personal characteristics that influence exercise behavior of older adults. $J$ Rehabil Res Dev. 2002; 39:95.

18. Olowookere AJ, Olowookere SA, Talabi AO, Etonyeaku AC, Adeleke OE, Akinboboye OO. Perceived family support and factors influencing medication adherence among hypertensive patients attending a Nigerian tertiary hospital. Ann Trop Med Public Health. 2015; 8:241.
19. Chawla N, Collins S, Bowen S, et al. The mindfulness-based relapse prevention adherence and competence scale: development, interrater reliability, and validity. Psychother Res. 2010;20:388-397.

20. Roebuck MC, Liberman JN, Gemmill-Toyama M, Brennan TA. Medication adherence leads to lower health care use and costs despite increased drug spending. Health Affairs. 2011;30:91-99.

21. Clark S, Farrington K, Chilcot J. Nonadherence in dialysis patients: prevalence, measurement, outcome, and psychological determinants. Semin Dial. 2014;27:42-49

22. Rolley JX, Davidson PM, Dennison CR, Ong A, Everett B, Salamonson Y. Centers for disease control and prevention. Chronic diseases and health promotion. 2008. http://www.cdc.gov/NCCdphp/ overview.htm. Accessed January 29, 2018.

23. Milchak JL, Carter BL, Ardery G, et al. Development of explicit criteria to measure adherence to hypertension guidelines. J Hum Hypertens. 2006; 20:426-433

24. Rich A, Brandes K, Mullan B, Hagger MS. Theory of planned behavior and adherence in chronic illness: a meta-analysis. J Behav Med. 2015;38(4): 673-688.

25. Armitage CJ, Conner M. Efficacy of the theory of planned behavior: a meta-analytic review. $\mathrm{Br} J$ Soc Psychol. 2001;40:471-499.

26. Gipson P, King C. Health behavior theories and research: implications for suicidal individuals' treatment linkage and adherence. Cogn Behav Pract. 2012;19:209-217.

27. Walker LO, Avant KC. Strategies for Theory Construct in Nursing (5th ed). New Jersey: PrenticeHall; 2011. 